\title{
Risk assessment in patients with systemic sclerosis and pulmonary arterial hypertension
}

\author{
Karen M. Olsson and Marius M. Hoeper \\ Affiliation: Dept of Respiratory Medicine, Hannover Medical School and German Center of Lung Research \\ (DZL), Hannover, Germany. \\ Correspondence: Marius M. Hoeper, Dept of Respiratory Medicine, Hannover Medical School, 30623 \\ Hannover, Germany. E-mail: hoeper.mariusamh-hannover.de
}

@ERSpublications

An abbreviated version of the ESC/ERS risk stratification strategy predicts outcome in patients with systemic sclerosis and pulmonary arterial hypertension http://ow.ly/4Zvh30lYJf3

Cite this article as: Olsson KM, Hoeper MM. Risk assessment in patients with systemic sclerosis and pulmonary arterial hypertension. Eur Respir J 2018; 52: 1801745 [https://doi.org/10.1183/13993003.017452018].

The current treatment of patients with pulmonary arterial hypertension $(\mathrm{PAH})$ is founded on repeated risk stratification at diagnosis and during follow-up. The 2015 European pulmonary hypertension guidelines propose a multidimensional risk assessment strategy based on nine items and 13 distinct variables in order to predict the estimated 1-year mortality $[1,2]$. Recently, several large registry studies have shown that simplified tools can be used to predict outcomes and guide treatment decisions [3-6]. These studies have focused largely on 6-min walking distance (6MWD), World Health Organization (WHO) functional class (FC), right atrial pressure (RAP), cardiac index (CI) and brain natriuretic peptides (BNP or NT-proBNP). Low-risk criteria were defined as WHO FC I or II, $6 \mathrm{MWD}>440 \mathrm{~m}, \mathrm{RAP}<8 \mathrm{mmHg}, \mathrm{CI} \geqslant 2.5 \mathrm{~L} \cdot \mathrm{min}^{-1} \cdot \mathrm{m}^{-2}$, BNP $<50 \mathrm{ng} \cdot \mathrm{L}^{-1}$ and NT-proBNP $<300 \mathrm{ng} \cdot \mathrm{L}^{-1}$. Risk stratification was based either on the average risk or the number of low-risk criteria. A unanimous finding of all these studies, irrespective of the assessment strategy, was that risk stratification at follow-up, i.e. in patients receiving $\mathrm{PAH}$ treatment, provided more accurate predictions of outcome than the initial risk assessment at the time of diagnosis [3-5, 7]. In addition, a noninvasive approach based solely on 6MWD, FC and BNP/NT-proBNP during follow-up was particular useful for identifying patients with a very low mortality risk ( $\leqslant 5 \%$ over a 5 -year observation period) $[4,8]$. Of note, these data were derived mainly from patients with idiopathic PAH (IPAH) and there was limited evidence that the same risk assessment strategy can be used in patients with other forms of $\mathrm{PAH}$, including those with PAH due to systemic sclerosis (SSc).

Although grouped together, SSc-PAH differs from IPAH in many aspects. Patients with SSc-PAH have a more complex disease often presenting with pulmonary and cardiac involvement, with extrapulmonary manifestations of their underlying disease and with more comorbidities. In addition, pulmonary vascular disease is not restricted to the precapillary vessels but includes rarefication of pulmonary capillaries and/or veno-occlusive disease $[9,10]$. These features may explain why response to PAH therapy and survival of patients with SSc-PAH are particularly poor $[11,12]$.

The present issue of the European Respiratory Journal contains two papers focusing on risk assessment in patients with SSc-PAH. In the first paper, Mercurio et al. [13] analysed 151 patients with SSc-PAH from the Johns Hopkins University. Follow-up data were available for 92 patients. The authors applied the same 
analytical strategies and used the same variables as previous studies. The authors mainly confirmed previous findings from studies in IPAH: 1) survival differed significantly among risk strata, regardless of the method of risk stratification; 2) the observed survival rates at 1-year were within the predicted ranges; and 3) improvements in risk category from baseline to the first follow-up assessment translated into better survival. At the same time, the study also showed that the overall survival at 5 years was just $40 \%$ and that only $22 \%$ of the patients were in the low risk category at follow-up.

The second paper comes from the French PAH Network registry and presents data from 513 patients with SSc-PAH. For 353 of these patients, follow-up data, including haemodynamics, were available from within the first year after diagnosis [14]. The overall 5-year survival rate was $35 \%$, i.e. as sobering as that found at Johns Hopkins; only $28 \%$ of the patients reached 3 or 4 low risk criteria at follow-up. As in the Johns Hopkins paper, mortality was significantly associated with the number of low-risk criteria at baseline and follow-up, again with a better prognostic performance of follow-up assessment.

The French investigators did not stop there, but further investigated a large set of haemodynamic variables in addition to RAP and CI, including stroke volume index (SVI), pulmonary vascular resistance (PVR) and pulmonary arterial compliance (PCa). As CI, SVI, PVR and PCa were highly correlated, separate multivariate modelling combining each single haemodynamic variable with 6MWD and WHO FC was performed. Among these haemodynamic variables, SVI was identified as the single most reliable predictor of outcome, confirming recent observations from the same group in patients with IPAH [15]. This finding is of particular importance as it suggests that CI should be replaced by SVI in future risk assessment strategies. This makes sense from a pathophysiological perspective, as SVI truly reflects contractile right ventricular function while CI is the product of heart rate and SVI, which means that CI can be maintained by an elevated heart rate despite a low SVI. In line with these considerations, $28 \%$ of patients from the French cohort with a CI $\geqslant 2.5 \mathrm{~L} \cdot \mathrm{min}^{-1} \cdot \mathrm{m}^{-2}$ had an SVI $<38 \mathrm{~mL} \cdot \mathrm{m}^{-2}$; these patients had a worse survival than patients with an SVI $\geqslant 38 \mathrm{~mL} \cdot \mathrm{m}^{-2}$.

Both the study from Johns Hopkins and the French study were limited by missing data, especially at follow-up. In the French series, this was particularly true for BNP/NT-proBNP, which was absent in almost half of the patients. In the subgroup of patients for whom these biomarkers were available, BNP/ NT-proBNP meeting low-risk criteria was associated with better transplant-free survival in the univariate analysis (HR $0.31,95 \%$ confidence interval $0.12-0.83 ; \mathrm{p}=0.02$ ), but not in the multivariate model. This stands in contrast with a previous study from the same group of researchers in patients with IPAH where NT-proBNP outperformed invasive haemodynamics, i.e. RAP and CI, in terms of prognostic value at follow-up [4]. In that study, both RAP and CI were independently associated with survival only as long as NT-proBNP was not part of the equation. These findings triggered an ongoing debate on the need and value of right heart catheterisation during follow-up $[16,17]$. The present data will fuel this discussion but leave several questions unanswered, including whether replacing CI by SVI will increase the predictive value of invasive haemodynamics in a clinically relevant manner, especially when NT-proBNP is available, and whether the prognostic information provided by invasive haemodynamics differs between patients with SSc-PAH and patients with IPAH.

Overall, the present papers clearly show that an abbreviated version of the ESC/ERS risk stratification can be utilised to predict the outcome of patients with SSc-PAH and guide treatment decisions, as has been demonstrated previously for patients with IPAH. The French study provides additional data that can be used to further improve risk prediction. At the same time, the outcome data from both studies remind us, once again, that the prognosis of patients with SSc-PAH remains poor, even when treated in highly skilled centres where all contemporary treatment options are available. Hence, improved risk prediction is welcome but what we need urgently are better treatment modalities for these patients.

Conflict of interest: K.M. Olsson reports personal fees for speaking and consultation from Actelion. M.M. Hoeper reports personal fees for speaking and consultation from Actelion, Bayer, GSK, Gilead and Merck.

\section{References}

1 Galie N, Humbert M, Vachiery JL, et al. 2015 ESC/ERS Guidelines for the diagnosis and treatment of pulmonary hypertension: The Joint Task Force for the Diagnosis and Treatment of Pulmonary Hypertension of the European Society of Cardiology (ESC) and the European Respiratory Society (ERS). Eur Respir J 2015; 46: 903-975.

2 Galie N, Humbert M, Vachiery JL, et al. 2015 ESC/ERS Guidelines for the diagnosis and treatment of pulmonary hypertension. Eur Heart J 2016; 37: 67-119.

3 Kylhammar D, Kjellstrom B, Hjalmarsson C, et al. A comprehensive risk stratification at early follow-up determines prognosis in pulmonary arterial hypertension. Eur Heart J 2017; in press [https://doi.org/10.1093/ eurheartj/ehx257]

4 Boucly A, Weatherald J, Savale L, et al. Risk assessment, prognosis and guideline implementation in pulmonary arterial hypertension. Eur Respir J 2017; 50: 1700889. 
5 Hoeper MM, Kramer T, Pan Z, et al. Mortality in pulmonary arterial hypertension: prediction by the 2015 European pulmonary hypertension guidelines risk stratification model. Eur Respir J 2017; 50: 1700740.

6 Hjalmarsson C, Radegran G, Kylhammar D, et al. Impact of age and comorbidity on risk stratification in idiopathic pulmonary arterial hypertension. Eur Respir J 2018; 51: 1702310.

7 Boucly A, Weatherald J, Humbert M, et al. Risk assessment in pulmonary arterial hypertension. Eur Respir J 2018; 51: 1800279 .

8 Hoeper MM, Pittrow D, Opitz C, et al. Risk assessment in pulmonary arterial hypertension. Eur Respir J 2018; 51: 1702606.

9 Dorfmuller P, Montani D, Humbert M. Beyond arterial remodelling: pulmonary venous and cardiac involvement in patients with systemic sclerosis-associated pulmonary arterial hypertension. Eur Respir J 2010; 35: 6-8.

10 Overbeek MJ, Vonk MC, Boonstra A, et al. Pulmonary arterial hypertension in limited cutaneous systemic sclerosis: a distinctive vasculopathy. Eur Respir J 2009; 34: 371-379.

11 Launay D, Sitbon O, Hachulla E, et al. Survival in systemic sclerosis-associated pulmonary arterial hypertension in the modern management era. Ann Rheum Dis 2013; 72: 1940-1946.

12 Condliffe R, Kiely DG, Peacock AJ, et al. Connective tissue disease-associated pulmonary arterial hypertension in the modern treatment era. Am J Respir Crit Care Med 2009; 179: 151-157.

13 Mercurio V, Diab N, Peloquin G, et al. Risk assessment in scleroderma patients with newly diagnosed pulmonary arterial hypertension: application of the ESC/ERS risk prediction model. Eur Respir J 2018; 52: 1800497.

14 Weatherald J, Boucly A, Launay D, et al. Haemodynamics and serial risk assessment in systemic sclerosis associated pulmonary arterial hypertension. Eur Respir J 2018; 52: 1800678.

15 Weatherald J, Boucly A, Chemla D, et al. Prognostic value of follow-up hemodynamic variables after initial management in pulmonary arterial hypertension. Circulation 2018; 137: 693-704.

16 Howard LS. Is right heart catheterisation still a fundamental part of the follow-up assessment of pulmonary arterial hypertension? The argument against. Eur Respir J 2018; 52: 1800996.

17 Humbert M, Weatherald J. Right heart catheterisation is still a fundamental part of the follow-up assessment of pulmonary arterial hypertension. Eur Respir J 2018; 52: 1800738. 